\title{
Achieving Data Completeness in Electronic Medical Records: A Conceptual Model and Hypotheses Development
}

\author{
Caihua Liu \\ University of Technology \\ Sydney \\ Caihua.Liu@uts.edu.au
}

\author{
Didar Zowghi \\ University of Technology \\ Sydney \\ Didar.Zowghi@uts.edu.au \\ Jay Daniel \\ University of Technology \\ Sydney \\ Jay.Daniel@uts.edu.au
}

\author{
Amir Talaei-Khoei \\ University of Nevada, Reno \\ atalaeikhoei@unr.edu
}

\begin{abstract}
This paper aims at proposing a conceptual model of achieving data completeness in electronic medical records (EMR). For this to happen, firstly, we draw on the model of factors influencing data quality management to construct our conceptual model. Secondly, we develop hypotheses of relationships between influencing factors for data completeness and mediators for achieving data completeness in EMR based on the literature. Our conceptual model extends the prior model for factors influencing data quality management by adding a new factor and exploring the relationships between the influencing factors within the context of data completeness in EMR. The proposed conceptual model and the presented hypotheses once empirically validated will be the basis for the development of tools and techniques for achieving data completeness in EMR.
\end{abstract}

\section{Introduction}

Most industries rely on their data for decision making. High data quality could assist organizations in working efficiently. However, incomplete data could increase additional scrutiny from those who use the data, because they have to exert more effort in dealing with missing data and interpreting the results of processing incomplete data [1]. As one dimension in data quality, data completeness has received extensive attention from data consumers. This is especially true in such a risk-averse industry such as healthcare, where decisions could literally determine life and death. Although data completeness has been the subject of much research in the last three decades, achieving data completeness for patient safety and quality of care remains as a significant challenge in healthcare. For example, it has been reported that less than $10 \%$ of medications approved by the Food and Drug Administration in Canada since 1980 have adequate data to analyze their risk for birth defects [2]. A large healthcare consortium fined $\$ 2.5$ million since they failed to turn over required data on patient care to the state's Medicaid program [3].

Problems associate with data incompleteness could result in severe consequences in healthcare such as increase in risks of patients' harm and loss of revenues. The importance of data completeness in healthcare has motivated us to conduct a research project on investigation of this phenomenon. The existing literature focusing on data completeness in electronic records seems to rely heavily on survey, case study and experiment, with only a few that have conceptualized factors influencing data completeness. Moreover, there is a paucity of studies that suggest specific concepts for achieving data completeness in electronic medical records (EMR). The underlying interactions between factors affecting the achievement of data completeness in EMR are unclear.

To address this gap in the literature, in this paper we aim to: (1) propose a conceptual model for achieving data completeness in EMR, (2) developing hypotheses for relationships between constructs affecting data completeness in EMR.

Our proposed conceptual model builds upon and extends the model for factors influencing data quality management [4] by adding a new factor and exploring the relationships between the influencing factors within the context of achieving data completeness in EMR. This study makes two contributions. First, we propose concepts of achieving data completeness in EMR that are lacking in the existing literature, developing 12 
hypotheses between influencing factors for data completeness and 11 mediators to achieve data completeness in EMR. Second, this study allows clinical staff to become more aware of data completeness in EMR at the point of care. The proposed model can serve as a tool for users in achieving data completeness in EMR.

The rest of this paper is organized as follows: Section 2 describes the concept of data completeness; Section 3 provides a brief theoretical background about related studies; Section 4 develops the hypotheses of our conceptual model; Section 5 outlines the construct operationalization; Section 6 presents discussion and conclusion of this study and our future work.

\section{Data completeness as a dimension of data quality}

As one dimension in data quality, data completeness refers to the extent to which all necessary data for specific activities is available for data users, providing accessible data that meets users' requirements for a given task. The general theory of data quality can be utilised to study data completeness and therefore, we take advantages of prior work on factors influencing data quality to construct our conceptual model. Moreover, our conceptual model could drive new insights into the achievement of data completeness in EMR.

\section{Theoretical support}

Prior studies have presented conceptual models for factors affecting data quality and empirically examined those models [4-10]. It is observed that the theory of data warehousing success $[5,6,10]$ and quality management theory [4, 7-10] (such as critical success factors for total quality management) contribute to understanding data quality management and improvement. We propose to follow the implementation of a related model [4] to construct our conceptual model within the context of achieving data completeness in EMR.

\subsection{Review of existing models}

A summary of characteristics on current dominant models of influencing factors for data quality is presented in Table 1 . We argue that these characteristics address three significant perspectives, including breadth, depth, and interaction of influencing factors for data quality that have been studied.
Table 1. A summary of characteristics on prior models for factors influencing data quality

\begin{tabular}{|c|c|c|c|c|c|c|c|c|}
\hline \multirow[t]{2}{*}{ Author(s)/Reference } & \multirow[t]{2}{*}{ 包 } & \multicolumn{4}{|c|}{ 苞 } & \multicolumn{2}{|c|}{ 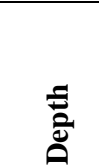 } & \multirow{2}{*}{ 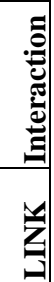 } \\
\hline & & $\mathbf{I}$ & $\sum$ & sy & 氠 & $\overrightarrow{\mathbf{Z}}$ & $\begin{array}{l}0 \\
0 \\
0 \\
0\end{array}$ & \\
\hline $\begin{array}{l}\text { Wixom and Watson } 2001 \\
{[6]}\end{array}$ & & $\mathrm{X}$ & $\mathrm{X}$ & $\mathrm{X}$ & & $\mathrm{X}$ & $x$ & \\
\hline Xu et al. 2002 [9] & & $\mathrm{x}$ & $\mathrm{x}$ & $\mathrm{x}$ & $\mathrm{x}$ & $\mathrm{x}$ & $\mathrm{x}$ & \\
\hline Nord et al. 2005 [8] & $\mathrm{x}$ & $\mathrm{x}$ & $\mathrm{x}$ & $\mathrm{x}$ & $\mathrm{x}$ & $\mathrm{x}$ & $\mathrm{x}$ & \\
\hline Tee et al. 2007 [10] & & $\mathrm{x}$ & $\mathrm{x}$ & $\mathrm{x}$ & $\mathrm{x}$ & & $\mathrm{x}$ & $\mathrm{x}$ \\
\hline Xiao et al. 2009 [4] & & $\mathrm{x}$ & $\mathrm{X}$ & $\mathrm{x}$ & & $\mathrm{x}$ & $\mathrm{X}$ & $\mathrm{X}$ \\
\hline Kokemueller 2011 [5] & $\mathrm{x}$ & $\mathrm{x}$ & $\mathrm{x}$ & $\mathrm{x}$ & & & $\mathrm{x}$ & $\mathrm{x}$ \\
\hline Xu 2013 [7] & $\mathrm{x}$ & $\mathrm{x}$ & $\mathrm{x}$ & $\mathrm{x}$ & $\mathrm{x}$ & $\mathrm{x}$ & $\mathrm{X}$ & \\
\hline \multicolumn{9}{|c|}{$\begin{array}{l}\text { CONT: The model is developed based on prior validated } \\
\text { concepts. } \\
\text { HF: Human factors affecting data quality } \\
\text { MF: Managerial factors affecting data quality } \\
\text { TF: Technical factors affecting data quality } \\
\text { EF: External factors affecting data quality } \\
\text { LINK: The model establishes relationships between } \\
\text { factors for data quality. } \\
\text { INDL: The model addresses factors at individual level. } \\
\text { ORGL: The model addresses factors at organizational } \\
\text { level. }\end{array}$} \\
\hline
\end{tabular}

First, breadth perspective refers to the extent to which factors affecting data quality were included in the conceptual model. Essentially, factors affecting data quality can be categorized into three groups: human, management and technology [4]. The human factors involve individual cognition and capability on data quality activities and the subjects could be decision-makers and/or staff members. The managerial factors concern the organization and coordination of the activities in an organization to achieve the defined goals of data quality, while technical factors contain existing electronic equipment, information technologies and systems to support the achievement of data quality. Meanwhile, many researchers revealed that external factors have an impact on data quality [7-9]. The external factors refer to forces outside an organization that have the potential to affect data quality such as physical [7-9] and legal changes [10]. Hence a more complete list of factors affecting data quality includes four main groups: human, managerial, technical, and external factors, as shown in Table 1. We can see that the model of critical success factors for data quality [79] gives a holistic picture of this subject targeting highest breadth, however, it is lacking interactions between these factors. 
Second, depth perspective concerns influencing factors for data quality that were studied from top to down in organizations. In other words, the commitment to data quality activities should address both at organizational and individual level. A body of literature indicates that commitment of top management is a key contributor to quality management $[4,8,9,11]$. Top management team needs to make many decisions on regulations and processes management and resources allocation in order to achieve goals of data quality [5]. At the same time, active engagement of staff members plays an essential role in the success of quality management [12], since personal competency and attitude determines the extent to which the tasks in relation to data quality can be completed [4]. Accordingly, both individual and organizational level (also depth) should be considered when studying factors affecting data quality.

Third, interaction perspective addresses relationships between influencing factors for data quality. Researchers have empirically examined relationships between influencing factors in their proposed model and indicated that these influencing factors for data quality dynamically relate to each other $[4,5,10]$. The underlying mechanisms of interactions between factors affecting data quality could help us potentially preserve quality data or systematically reduce bad data. Thus, establishment of links between factors contributing to data quality is a significant enabler to ensure quality data. Although external factors were not included in Xiao et al.'s work [4], they developed a stronger conceptual model for factors influencing data quality with richness and reach in breadth, depth, and interaction.

Based upon this analysis, we construct the conceptual model on the model of factors influencing data quality management [4], while combining the strengths of those models [4, 5] and considering breadth, depth, and interaction perspective for factors contributing to achieving data completeness in EMR.

\subsection{Utilizing the model of factors influencing data quality management in achieving data completeness in EMR}

Using the theory of product quality management, Xiao et al. [4] provided a systematic discussion about influencing factors for data quality management. These findings, together with organisational behaviour theory, drove Xiao et al. [4] to propose a model of factors affecting data quality management within 3 perspectives (strategic, management and infrastructure) and 5 factors that can be grouped into different perspectives. These five factors are top management support (TMS), capability on the regulation and process management (CRPM), business-IT alignment (BITA), staff participation (SP), and integration of IS (IIS). Moreover, they empirically examined relationships between these factors. Figure 1 presents the model of factors influencing data quality management within significant relations between the influencing factors.

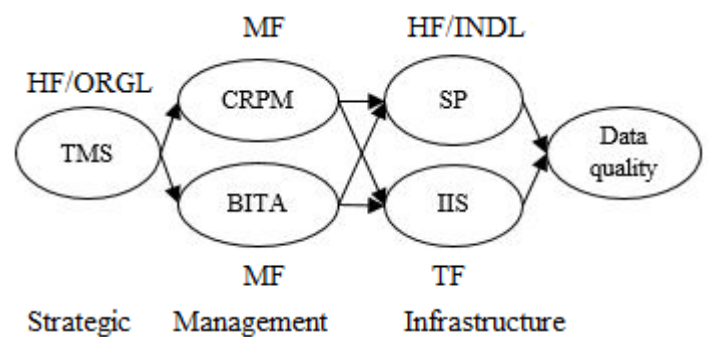

Figure 1. The model of factors influencing data quality management taken from Xiao et al.'s work [4]

In this work, we aim to develop a conceptual model for achieving data completeness in EMR. Xiao et al. [4] offered a guideline within breadth, depth, and interaction of influencing factors for data quality, addressing process of managing data quality from strategic, management and infrastructure perspectives. Furthermore, the existing literature focusing on data completeness in electronic records could gain rich insights of relationships between factors affecting data completeness achievement in EMR that are described in the following section.

\section{Hypotheses development and literature support}

To construct our conceptual model, the literature of data completeness in electronic records, data quality management, quality management was reviewed. These findings, together with a set of factors affecting data quality in different IS and data warehouses, were used to create our conceptual model for achieving data completeness in EMR and develop hypotheses of the model.

Here, human factors involve clinic director and clinical staff. For managerial factors, appropriate regulation formulation, process management, and resources allocation for EMR implementation could help staff members achieve better data completeness in EMR, while EMR integration provides technical support that has an impact on achieving data completeness in EMR.

We now start with the model of factors influencing data quality management [4] and retain significant relations between these influencing factors. Due to 
significant impact of Resources on Organizational Implementation Success for improving data quality [5] that reflects capability of regulation and process management in an organization, we also add Resources factor into our resulting conceptual model (see Figure 2 ). We use dotted lines to highlight new factor and relationships added in the proposed model. Our conceptual model for achieving data completeness in EMR extends the model from Xiao et al. [4] in the following ways:

(i) New factors added: Resources

(ii) New relationships added:

- Resources and Regulatory capability for EMRenabled care processes

- Resources and EMR alignment to care processes

- Resources and EMR integration

- Regulatory capability for EMR-enabled care processes and Data completeness in EMR

The factors affecting the achievement of data completeness in EMR in our conceptual model address both system design and implementation as well as system use post implementation. Four factors are used in the stage of system design and implementation including Clinic director's support for EMR implementation, Resources, Regulatory capability for EMR-enabled care processes, and EMR alignment to care processes. Two factors are defined in the stage of system use post implementation concerning Clinical staff's participation and EMR integration. In this light, our conceptual model can address the relevant controls from both system design and implementation as well as system use post implementation to guarantee data completeness in EMR.

The rationale for relationships among influencing factors for achieving data completeness in EMR are described below.
4.1. Relationship between clinic director's support for EMR implementation for data completeness with regulatory capability for EMR-enabled care processes to achieve data completeness in EMR

Clinic director's support for EMR implementation refers to the extent to which upper management level in clinics understands the importance of EMR implementation and participants in the relevant activities. Prior studies have asserted that top management commitment has a positive impact on the regulation and process management for IS implementation, because decision makers' attitude and knowledge about innovative IS and data quality initiatives determines the degree to which resources can be allocated $[5,6]$ and political resistance to the implementation of systems can be dealt with [6].

Similarly, the management of EMR-enabled care processes for achieving data completeness in EMR cannot ignore support from the clinic director who is accountable for the entire running of the clinic. We hypothesize:

$H_{1}$ : A high level of clinic director's support for EMR implementation is positively associated with regulatory capability for EMR-enabled care processes for achieving data completeness in EMR.

\subsection{Relationship between clinic director's support for EMR implementation for data completeness with EMR alignment to care processes to achieve data completeness in EMR}

The implementation of IS in organizations includes a process of business-IT alignment [4]. During this process, decision makers need to carry out critical decisions and coordination for achieving alignment

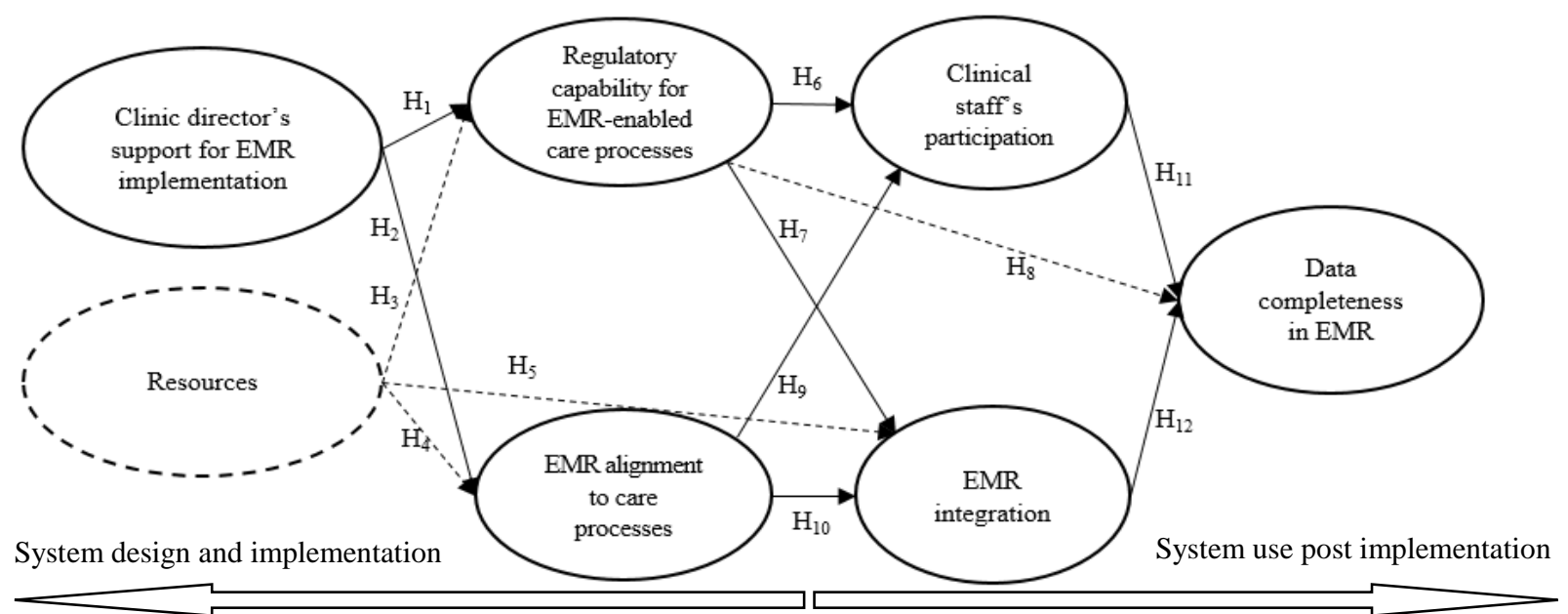

Figure 2. The conceptual model of achieving data completeness in EMR proposed in this study 
between business and IT. Here, the EMR implementation could change the existing work processes that may introduce barriers to its adoption [13]. A good understanding and knowledge about EMR systems and supporting technologies could drive the clinic director to make commitment to communication and coordination between IT and clinical staff for EMR implementation and to locate required resources for EMR implementation in care processes. As a result, the alignment between EMR and care processes could be facilitated that help achieve data completeness in EMR. We hypothesize:

$\mathrm{H}_{2}$ : A high level of clinic director's support for EMR implementation is positively associated with EMR alignment to care processes for achieving data completeness in EMR.

\subsection{Relationship between resources for data completeness with regulatory capability for EMR-enabled care processes to achieve data completeness in EMR}

Resources in our conceptual model include funding, human resource, and time for EMR implementation.

Resources are important to IT implementation, since these activities are time-consuming and expensive $[5,6]$. Due to unclear return on investment in the short term but large investment in technology, the EMR carries barriers to its implementation for care [14]. Accordingly, resources are vital for successful implementation of EMR-enabled care processes. The sufficient human resource, available time and funding being allocated to EMR implementation, could guarantee effective technology being used for the EMR implementation. Without these efforts, EMR implementation integrated into care processes and relevant data completeness activities are unlikely to conduct. For example, database specialists' time is not made available to these initiatives and therefore, EMRenabled care processes could be delayed, which has an impact on achieving data completeness in EMR. We hypothesize:

$\mathrm{H}_{3}$ : A high level of resources is positively associated with regulatory capability for EMR-enabled care processes for achieving data completeness in EMR.

\subsection{Relationship between resources for data completeness with EMR alignment to care processes to achieve data completeness in EMR}

EMR alignment to care processes involves communication and coordination about EMR implementation in the clinic between clinical and IT staff. Accordingly, investigators should capture users' requirements and data completeness problems by analyzing the current system and interviewing users [15]. At this time, a set of considerable resources are also in need of being addressed for organizing and supporting a network of users in this process [16]. We can see that resources have an impact on EMR alignment to care processes in order to achieve data completeness in EMR. We hypothesize:

$\mathrm{H}_{4}$ : A high level of resources is positively associated with EMR alignment to care processes for achieving data completeness in EMR.

\subsection{Relationship between resources for data completeness with EMR integration to achieve data completeness in EMR}

The EMR are implemented across clinical systems and repositories, providing a comprehensive view for a patient by integrating diverse data sources [17]. Here, the integration technologies (such as data warehouse) to some degree determine the extent to which the data is aggregated from different systems [18]. The EMR integration requires investment in technology as mentioned in Section 4.3. Thus, the clinic with skillful IT professionals and mature technology for EMR implementation could have better chance to aggregate complete data from various data sources. We hypothesize:

$H_{5}$ : A high level of resources is positively associated with EMR integration for achieving data completeness in EMR.

\subsection{Relationship between regulatory capability for EMR-enabled care processes for data completeness with clinical staff's participation to achieve data completeness in EMR}

Regulatory capability for EMR-enabled care processes describes a clinic's ability to design, implement and improve (1) a set of rules and standards that regulate care processes when using EMR, and (2) a complete system of procedures for achieving the clinic's goals of data completeness in using EMR.

Based on structured rules and procedures in the use of IS [19-21], individuals are required to follow a set of regulations and processes for addressing data quality [4]. At this time, the clinic may conduct appropriate trainings to improve staff's ability of using EMR in the care processes. For example, the training of procedures for recording clinical information could enhance staff' skills for preventing data completeness issues at the point of care [22]. Furthermore, the emphasis on the importance of data completeness in record keeping 
tasks in training could help staff realize that incomplete data in clinical use could result in severe consequence at point of care. As a result, staff in the clinic would favorably follow the relevant operational policy and play their roles in data completeness activities when using EMR. Based on this analysis, we hypothesize:

$H_{6}$ : A high level of regulatory capability for EMRenabled care processes is positively associated with clinical staff's participation for achieving data completeness in EMR.

\subsection{Relationship between regulatory capability for EMR-enabled care processes for data completeness with EMR integration to achieve data completeness in EMR}

The implementation and optimization of regulation and process demand from higher IS integration, thereby furthering the integrated application of IS [4]. Here, the detailed management infrastructure, with a complete system and clear procedures for using EMR, could facilitate EMR integration. For instance, specific standard and process representations could help better understand the policy of data collection when using EMR for achieving complete data at the point of care [23]. In this way, IT professionals could better support EMR integration in order to deliver the corresponding information for different use. Therefore, we hypothesize:

$H_{7}:$ A high level of regulatory capability for EMRenabled care processes is positively associated with EMR integration for achieving data completeness in EMR.

\subsection{Relationship between regulatory capability for EMR-enabled care processes and data completeness in EMR}

Changes in routine management may emerge due to implementation of innovative IS, which could result in political resistance in organizations [24]. If practitioners can put change management programs in place, deal with political resistance effectively, and encourage organizational members to embrace these innovations, the implementation of IS could lead to a high level of data quality [6]. Here, EMR implementation could also result in changes in care processes, and at this time practitioners should consider countermeasures to addressing these challenges for EMR implementation. For instance, a structured process designed to deal with human factors through behavior change could assist to achieve the expected benefits of EMR implementation [25], thus having great potential to achieve data completeness in EMR. We hypothesize:

$H_{8}$ : A high level of regulatory capability for EMRenabled care processes is positively associated with achieving data completeness in EMR.

\subsection{Relationship between EMR alignment to care processes for data completeness with clinical staff's participation to achieve data completeness in EMR}

EMR alignment to care processes refers to communication and coordination about EMR implementation between clinical and IT staff. According to Total Data Quality Management methodology, organizations must first define what they mean by data quality and then establish a usable metrics linked to data stakeholders' goals for measuring how good is the data [26]. As a result, a good understanding and communication on the definition of data completeness in EMR from data users can serve as a foundation for clear alignment between intention of data creation and its usage. In this way, the EMR could offer the corresponding information to clinical staff at the point of care. These effective practices of achieving data completeness could increase awareness of maintenance and usage of the data in clinical decision making and planning of health services and products. Accordingly, clinical staff are motivated to further support data completeness initiatives when using EMR. We hypothesize:

$H_{9}:$ A high level of EMR alignment to care processes is positively associated with clinical staff's participation for achieving data completeness in EMR.

\subsection{Relationship between EMR alignment to care processes for data completeness with EMR integration to achieve data completeness in EMR}

An effective coordination and communication between business and IT can facilitate integration of IS [4]. Here, the clinical staff provide feedback about the utilization of EMR in their work processes, and IT professionals can capture users' voices so as to customize countermeasures to solving mismatch problems between workflow and technology in using EMR. The procedures of initiative IS integrated into the work processes could be accepted into organizations and facilitate the delivery of qualityassured services [27]. Accordingly, the understanding and coordination about EMR implementation between 
IT and clinical staff could serve as a foundation for EMR integration. In this way, the data in EMR aggregated from multiple clinical systems could achieve clinical staff's needs at the point of care. We hypothesize:

$H_{10}:$ A high level of EMR alignment to care processes is positively associated with EMR integration for achieving data completeness in EMR.

\subsection{Relationship between clinical staff's participation and data completeness in EMR}

Clinical staff's participation in EMR context is the process whereby employees are involved in running and improving clinical processes and data completeness activities when using EMR.

The misunderstanding or insufficient knowledge about data entry policy could result in incomplete documentation [23]. Furthermore, if a staff member lacks the awareness about importance of data recording or reporting tasks, data delays or omission errors might occur due to human carelessness [22]. In addition, the availability of dedicated time for dealing with recording tasks puts staff members under pressure [15, 22]. As a result, more missing items could emerge during the data entry. The data may not be available at the time where data needs to be entered in EMR. This unavailability of data will result in data incompleteness. In short, individual awareness, attitude, mental status and competency determine the degree of participating data completeness achievement when using EMR. We hypothesize:

$H_{11}:$ A high level of clinical staff's participation is positively associated with achieving data completeness in EMR.

\subsection{Relationship between EMR integration and data completeness in EMR}

EMR integration describes that the EMR are implemented across clinical systems and repositories, providing integrated data for different needs.

The architecture of the EMR is a formal description of a system, organizing components and services that support recording, retrieving and handling information in EMR [17]. The EMR are scattered across multiple clinical systems and repositories for patient-centred continuity of care [17]. Using the EMR, nurses can follow up processes of care and physicians can check results of tests for clinical decision making. An EMR can serve for different use within integrated data since a subsystem cannot provide a complete picture of a patient's condition to clinical staff [28]. As mentioned in Section 4.5, integration technologies could determine the extent to which the data can be aggregated from different systems. Accordingly, the extent to EMR integration could ascertain how good is the data in the EMR. We hypothesize:

$H_{12}$ : A high level of EMR integration is positively associated with achieving data completeness in EMR.

\subsection{Mediators in achieving data completeness in EMR}

We have proposed 12 hypotheses about relationships between the influencing factors and data completeness in EMR (see Figure 2). Accordingly, there are 11 mediators in achieving data completeness in EMR posited in our conceptual model, as shown in Table 2.

Table 2. Hypotheses of mediators for achieving data completeness in EMR posited in the conceptual model

$\mathrm{H}_{13}$ : Mediator 1 of achieving data completeness in EMR: Clinic director's support for EMR implementation Regulatory capability for EMR-enabled care processes Data completeness in EMR

$\mathrm{H}_{14}$ : Mediator2 of achieving data completeness in EMR: Clinic director's support for EMR implementation Regulatory capability for EMR-enabled care processes Clinical staff's participation - Data completeness in EMR $\mathrm{H}_{15}$ : Mediator 3 of achieving data completeness in EMR: Clinic director's support for EMR implementation Regulatory capability for EMR-enabled care processes EMR integration - Data completeness in EMR

$\mathrm{H}_{16}$ : Mediator 4 of achieving data completeness in EMR: Clinic director's support for EMR implementation EMR alignment to care processes - Clinical staff's participation - Data completeness in EMR

$\mathrm{H}_{17}$ : Mediator5 of achieving data completeness in EMR: Clinic director's support for EMR implementation EMR alignment to care processes - EMR integration Data completeness in EMR

$\mathrm{H}_{18}$ : Mediator 6 of achieving data completeness in EMR: Resources - Regulatory capability for EMR-enabled care processes - Clinical staff's participation - Data completeness in EMR

$\mathrm{H}_{19}$ : Mediator 7 of achieving data completeness in EMR: Resources -Regulatory capability for EMR-enabled care processes - Data completeness in EMR

$\mathrm{H}_{20}$ : Mediator8 of achieving data completeness in EMR: Resources - Regulatory capability for EMR-enabled care processes - EMR Integration - Data completeness in EMR $\mathrm{H}_{21}$ : Mediator9 of achieving data completeness in EMR: Resources - EMR integration - Data completeness in EMR $\mathrm{H}_{22}$ : Mediator 10 of achieving data completeness in EMR: Resources - EMR alignment to care processes - Clinical staff's participation - Data completeness in EMR

$\mathrm{H}_{23}$ : Mediator ${ }_{11}$ of achieving data completeness in EMR: Resources - EMR alignment to care processes - EMR integration - Data completeness in EMR 


\section{Construct operationalization}

We intend to operationalize our constructs based on their measurement (in Appendix A) in our research project. Since data completeness implies that the data describes all the facts in the real world, complete data should be accurate and up-to-date as well. In other words, data completeness overlaps with other dimensions in data quality such as accuracy [29, 30]. Furthermore, this construct includes system design and implementation as well as system use post implementation factors that link clinic director's support for EMR implementation and resources with achieving data completeness in EMR. For system design and implementation factors, clinic director's support for EMR implementation measures clinic director's awareness, attitude, and competency towards activities concerning EMR implementation, while factor about resources measures funding, human resource, and time for the implementation. Regulatory capability for EMRenabled care processes measures a clinic's ability to design, implement, and improve data quality activities and process management of EMR implementation, while EMR alignment to care processes measures the level of communication and collaboration in care processes and data completeness issues about EMR implementation between clinical and IT staff. For system use post implementation factors, clinical staff's participation measures awareness, attitude, competency and mental status of clinical staff towards data completeness activities in using EMR, while EMR integration measures the quality of the EMR system including ease of use, usefulness, and compatibility. The definition and sources of these measures in each factor are presented in Appendix A in details.

\section{Discussion, conclusion and future studies}

This paper utilizes prior models for factors affecting data quality in IS and data warehouses and draws on the model of factors contributing to data quality management [4] (see Section 3), to propose a conceptual model within the context of achieving data completeness in EMR. Additionally, the literature on data completeness in electronic records, data quality management and quality management was reviewed and synthesized to propose 12 hypotheses for the influencing factors and 11 mediators to achieve data completeness in EMR (as presented in Section 4). Our work makes two important contributions as presented below.

\subsection{Theoretical contributions}

First, the identification of influencing factors for achieving data completeness in EMR in this work provides an initial explanation of this phenomenon. We construct a conceptual model for achieving data completeness in EMR, including hypotheses development of relationships between influencing factors for data completeness and mediators to achieve data completeness in EMR, which is lacking in the existing literature. Second, our conceptual model aligns well with breadth, depth and interaction perspective to study influencing factors for achieving data completeness in EMR. We extend prior model of factors influencing data quality management within the context of EMR by adding one new factor and the relationships between the influencing factors.

Accordingly, we posit that other data quality problems also can be studied in a similar way. This study therefore encourages academics to (1) take advantages of inductive methods in the identification of practices that address other data quality problems in EMR; and (2) adopt deductive methods to validate conceptual model.

\subsection{Practical contributions}

Understanding influencing factors for achieving data completeness in EMR allows practitioners to become more aware of issues that need to be taken into consideration. The proposed model can be utilised as a tool by data consumers for achieving completeness in EMR. In order to achieve data completeness in EMR, this study suggests that practitioners should pay attention to the following issues. First, the metrics of completeness must be defined based on users' requirements. The alignment between intention of data creation and its usage could serve as a basis for delivery of corresponding information products to users for specific tasks. Second, data completeness is a complicated concept and its problems could emerge at any point from data entry to data analysis and decision making. Effective organizational and technical strategies for achievement of data completeness in EMR could enhance a clinical staff's capability for achieving quality of clinical decision making and quality of care.

The present study serves as a conceptual paper investigating the literature and theoretical support to improve data completeness in EMRs. Although we acknowledge the lack of data to support the argument presented in this paper, we plan to collect data and generate empirical results to accept or reject the hypothetical claims in this study.

Accordingly, our future work will empirically examine the proposed conceptual model and the developed hypotheses through surveys and a case study as part of 
the overall research project. The targeted subjects will involve physicians, nurses, medical informatics experts, and IT professionals who have backgrounds or experience of dealing with the EMR data in Australia.

\section{References}

[1] B. E. Dixon, J. J. McGowan, and S. J. Grannis, "Electronic laboratory data quality and the value of a health information exchange to support public health reporting processes," in AMIA Annu Symp Proc., 2011, pp. 322-330.

[2] M. P. Adam, J. E. Polifka, and J. Friedman, "Evolving knowledge of the teratogenicity of medications in human pregnancy," American Journal of Medical Genetics Part C: Seminars in Medical Genetics, vol. 157C, no. 3, pp. 175-182, 2011.

[3] C. Terhune. (2017, January 26). California Fines Kaiser Permanente \$2.5 Million Over Missing Medicaid Data. Kaiser Health News. Retrieved May 16, 2017, from: http://khn.org/news/california-fines-kaiser-permanente-2-5million-over-missing-medicaid-data/

[4] J.-H. Xiao, K. Xie, and X.-W. Wan, "Factors influencing enterprise to improve data quality in information systems application -An empirical research on 185 enterprises through field study," in 2009 International Conference on Management Science and Engineering, 2009, pp. 23-33.

[5] J. Kokemueller, "An empirical investigation of factors influencing data quality improvement success," in AMCIS 2011 Proc., 2011.

[6] B. H. Wixom and H. J. Watson, "An empirical investigation of the factors affecting data warehousing success," MIS Quarterly, vol. 25, no. 1, pp. 17-41, 2001.

[7] H.-J. Xu, "Factor analysis of critical success factors for data quality," in AMCIS 2013 Proc., 2013.

[8] G. D. Nord, J. H. Nord, and H. Xu, "An investigation of the impact of organization size on data quality issues," Journal of Database Management, vol. 16, pp. 58-71, 2005.

[9] H.-J. Xu, A. Koronius, and N. Brown, "Managing data quality in accounting information systems," IT-Based Management: Challenges and Solutions, pp. 277-299, 2002.

[10] S. W. Tee, P. L. Bowen, P. Doyle, and F. H. Rohde, "Factors influencing organizations to improve data quality in their information systems," Accounting \& Finance, vol. 47, pp. 335-355, 2007.

[11] N. Zellal and A. Zaouia, "An exploratory investigation of factors influencing data quality in data warehouse," in 2015 Third World Conference on Complex Systems, 2015, pp. 1-6.

[12] R. B. Sharma, Total Quality Management. John Wiley \& Sons Ltd., 2015.
[13] C. Lin, I.-C. Lin, and J. Roan, "Barriers to physicians' adoption of healthcare information technology: an empirical study on multiple hospitals," Journal of Medical Systems, vol. 36, pp. 1965-1977, 2012.

[14] J. Adler-Milstein, A. P. McAfee, D. W. Bates, and A. $\mathrm{K}$. Jha, "The state of regional health information organizations: current activities and financing," Health Affairs, vol. 27, pp. w60-w69, 2008.

[15] M. Staff, C. Roberts, and L. March, "The completeness of electronic medical record data for patients with Type 2 Diabetes in primary care and its implications for computer modelling of predicted clinical outcomes," Primary Care Diabetes, vol. 10, pp. 352-359, 2016.

[16] L. Damodaran, "User involvement in the systems design process-a practical guide for users," Behaviour \& Information Technology, vol. 15, pp. 363-377, 1996.

[17] ISO. (2011, 21 March). Health informatics - Requirements for an electronic health record architecture. Retrieved May 30, 2017, from: https://www.iso.org/obp/ui/\#iso:std:iso:18308:ed-1:v1:en

[18] C. Cappiello, C. Francalanci, and B. Pernici, "Timerelated factors of data quality in multichannel information systems," Journal of Management Information Systems, vol. 20, pp. 71-92, 2003.

[19] S. C. Sigei, Critical Success Factors in the Implementation of the Re-engineered Integrated Financial Management Information System in Government Ministries, Kenya. University of Nairobi, 2013.

[20] T. Puschmann and R. Alt, "Enterprise application integration systems and architecture-the case of the Robert Bosch Group," Journal of Enterprise Information Management, vol. 17, pp. 105-116, 2004.

[21] M. M. Yusof, J. Kuljis, A. Papazafeiropoulou, and L. K. Stergioulas, "An evaluation framework for Health Information Systems: human, organization and technology-fit factors (HOT-fit)," International Journal of Medical Informatics, vol. 77, pp. 386-398, 2008.

[22] A. Warsi, S. White, and P. McCulloch, "Completeness of data entry in three cancer surgery databases," European Journal of Surgical Oncology, vol. 28, pp. 850-856, 2002.

[23] R. R. Kelley, W. A. Mattingly, T. L. Wiemken, M. Khan, D. Coats, D. Curran, et al., "Visual grids for managing data completeness in clinical research datasets," Journal of Biomedical Informatics, vol. 54, pp. 337-344, 2015.

[24] M. Pardo del Val and C. Martínez Fuentes, "Resistance to change: a literature review and empirical study," Management Decision, vol. 41, pp. 148-155, 2003.

[25] C. McCarthy and D. Eastman, Change Management Strategies for an Effective EMR Implementation. Chicago: HIMSS, 2010. 
[26] R. Y. Wang, "A product perspective on total data quality management," Communications of the ACM, vol. 41, pp. 58$65,1998$.

[27] S. Herzberg, K. Rahbar, L. Stegger, M. Schäfers, and M. Dugas, "Concept and implementation of a computerbased reminder system to increase completeness in clinical documentation," International Journal of Medical Informatics, vol. 80, pp. 351-358, 2011.

[28] National Institutes of Health, National Center for Research Resources (2006, March 23). Electronic Health Records Overview. Retrieved May 30, 2017, from: http://www.himss.org/electronic-health-records-overviewnih-national-center-research-resources

[29] C. Liu, A. Talaei-Khoei, D. Zowghi \& J. Daniel, "Data completeness in healthcare: a literature survey", Pacific Asia Journal of the Association for Information Systems, vol. 9, no. 2, pp.75-100, 2017.
[30] N. G. Weiskopf and C. Weng, "Methods and dimensions of electronic health record data quality assessment: enabling reuse for clinical research," Journal of the American Medical Informatics Association, vol. 20, pp. 144-151, 2013.

[31] J. Juran and A. B. Godfrey, Juran's Quality Handbook. NY: McGraw-Hill, 1999.

[32] F. Köpcke, B. Trinczek, R. W. Majeed, B. Schreiweis, J. Wenk, T. Leusch, et al., "Evaluation of data completeness in the electronic health record for the purpose of patient recruitment into clinical trials: a retrospective analysis of element presence," BMC Medical Informatics and Decision Making, vol. 13, p. 37, 2013.

[33] J. Jang, S. H. Yu, C.-B. Kim, Y. Moon, and S. Kim, "The effects of an electronic medical record on the completeness of documentation in the anesthesia record," International Journal of Medical Informatics, vol. 82, pp. 702-707, 2013.

\section{Appendix A. Evaluation measures and their definitions in our proposed model}

\begin{tabular}{|c|c|c|}
\hline $\begin{array}{l}\text { Construct } \\
\text { factors }\end{array}$ & $\begin{array}{l}\text { Evaluation } \\
\text { measures }\end{array}$ & Definition \\
\hline \multirow{3}{*}{ Resources } & Funding & $\begin{array}{l}\text { Budgets are available to successfully complete the tasks related to EMR } \\
\text { implementation and data completeness initiatives (revised from prior work }[5,6] \text { ). }\end{array}$ \\
\hline & Human resource & $\begin{array}{l}\text { People are available to successfully complete the tasks related to EMR implementation } \\
\text { and data completeness initiatives (revised from prior work }[5,6] \text { ). }\end{array}$ \\
\hline & Time & $\begin{array}{l}\text { Time is available to successfully complete the tasks related to EMR implementation } \\
\text { and data completeness initiatives (revised from prior work }[5,6] \text { ). }\end{array}$ \\
\hline \multirow{3}{*}{$\begin{array}{l}\text { Clinic director's } \\
\text { support for } \\
\text { EMR } \\
\text { implementation }\end{array}$} & Awareness & $\begin{array}{l}\text { Clinic director recognizes importance of EMR implementation and data completeness } \\
\text { in EMR (revised from prior work }[4,7,8,10] \text { ). }\end{array}$ \\
\hline & Attitude & Clinic director supports EMR implementation and data completeness activities [4-10]. \\
\hline & Competency & $\begin{array}{l}\text { Competency of clinic director describes leadership skills and knowledge of EMR } \\
\text { values (revised from prior work [4]). }\end{array}$ \\
\hline \multirow{2}{*}{$\begin{array}{l}\text { Regulatory } \\
\text { capability of } \\
\text { EMR-enabled } \\
\text { care processes }\end{array}$} & $\begin{array}{l}\text { Data quality } \\
\text { management }\end{array}$ & $\begin{array}{l}\text { This concerns a set of data quality activities such as data quality planning, regulation } \\
\text { and controls (revised from Xu et al.'s work [7]). }\end{array}$ \\
\hline & $\begin{array}{l}\text { Process } \\
\text { management }\end{array}$ & $\begin{array}{l}\text { Process management can be viewed as the sum total of the ways in which it involves } \\
\text { the information chains from data acquisition to its usage during EMR-enabled care } \\
\text { processes (revised from prior work [31]). }\end{array}$ \\
\hline \multirow{2}{*}{$\begin{array}{l}\text { EMR alignment } \\
\text { to care } \\
\text { processes }\end{array}$} & Alignment & $\begin{array}{l}\text { The EMR system works well with routine operation in an organization (revised from } \\
\text { Xiao et al.'s work [4]). }\end{array}$ \\
\hline & Communication & $\begin{array}{l}\text { Communication and collaboration about data completeness issues in EMR between IT } \\
\text { professionals and clinical staff works effectively (revised from Xiao et al.'s work [4]). }\end{array}$ \\
\hline \multirow{4}{*}{$\begin{array}{l}\text { Clinical staff's } \\
\text { participation }\end{array}$} & Awareness & $\begin{array}{l}\text { Clinical staff recognize the importance of data completeness in EMR (revised from } \\
\text { prior work }[4,7,8,22]) \text {. }\end{array}$ \\
\hline & Attitude & Clinical staff support data quality activities (revised from prior work $[4,7,8,22]$ ). \\
\hline & Competency & $\begin{array}{l}\text { Competency of clinical staff describes knowledge and skills in dealing with EMR data } \\
{[15,23,32] \text {. }}\end{array}$ \\
\hline & Mental status & $\begin{array}{l}\text { Individual fatigue and tension could trigger human errors in achieving data } \\
\text { completeness in EMR }[22,33] \text {. }\end{array}$ \\
\hline \multirow{3}{*}{$\begin{array}{l}\text { EMR } \\
\text { Integration }\end{array}$} & Ease of use & $\begin{array}{l}\text { The EMR system is easy to use, such as unambiguous design of user interface (revised } \\
\text { from prior work [15]). }\end{array}$ \\
\hline & Usefulness & $\begin{array}{l}\text { The EMR system effectively integrated data from different functional areas and } \\
\text { addresses data needs as clinical staff arise (revised from prior work }[5,6] \text { ). }\end{array}$ \\
\hline & Compatibility & $\begin{array}{l}\text { The implementation of EMR system overcomes numerous technical problems and } \\
\text { works well across multiple clinical systems and repositories (revised from prior work } \\
{[5,6] \text { ). }}\end{array}$ \\
\hline \multirow{2}{*}{$\begin{array}{l}\text { Data } \\
\text { completeness in } \\
\text { EMR }\end{array}$} & Availability & $\begin{array}{l}\text { The required data is available for users when using the EMR system (revised from prior } \\
\text { work [30]). }\end{array}$ \\
\hline & Accuracy & The data provided by the EMR system is accurate (revised from prior work [30]). \\
\hline
\end{tabular}

